RESEARCHARTICLE

\section{Self-Compassion Differences in Women Who Have Experienced Sexual Assault and Nonsexual Assault Trauma}

\author{
Jessica R. Williamson* \\ California State University, Bakersfield, USA
}

\begin{abstract}
In the current exploratory study, differences in self-compassion were examined for women who listed experiencing sexual assault related trauma and nonsexual assault trauma (e.g. violent death or injury of loved one, witnessing death, injury to self, natural disasters, acts of war). Results indicate that individuals who have experienced sexual assault report significantly lower levels of self-compassion compared to those who experience traumas that do not involve sexual assault. Examination of differences in subscale scores of the Self-Compassion scale revealed that those who experienced sexual assault were significantly higher in self-judgment, isolation, and overidentification and marginally significantly lower in self-kindness compared to individuals who experience other types of trauma. Implications for refined treatment approaches involving the inclusion of self-compassion inductions for women who experience sexual assault-related trauma are discussed.
\end{abstract}

\section{Keywords}

Self-compassion, trauma, sexual assault

\section{Introduction}

Open Access

Citation: Williamson, J.R. Self-Compassion Differences in Those Who Have Experienced Sexual Assault and Non-Sexual Assault Trauma. Gender and Women's Studies. 2019; 2(3):3.

Received: February 26, 2019

Accepted: July 26, 2019

Published: August 9, 2019

Copyright: @ 2019 Williamson J R. This is an open access article distributed under the terms of the Creative Commons Attribution License, which permits unrestricted use, distribution, and reproduction in any medium, provided the original author and source are credited.

\section{Corresponding author:} Jessica R. Williamson, California State University, Bakersfield, USA

E-mail:jessrwilliamson@gmail.com; Jwilliamson12@csub.edu
According to the Centers for Disease Control and Prevention (CDC, 2019; Basile, Smith, Breidling, Black, \& Mahendra, 2014; See also CDC report prepared by Black et al., 2011), 1 in 3 women have experienced sexual violence (including rape, sexual coercion, unwanted sexual contact, and noncontact unwanted sexual experiences) at some point in their lives. Rape (experienced by 1 in 5 women) is defined as any completed or attempted unwanted vaginal (for people with female biology), oral, or anal penetration through the use of physical force (such as being pinned or held down, or by the use of violence) or threats to physically harm and includes times when the victim was drunk, high, drugged, or passed out and unable to consent (CDC, 2019). Rape is separated into three types of events: Completed forced penetration, attempted forced penetration, and completed alcohol or drug facilitated penetration (Black et al., 2011, pp. 4). Black et al. (2011) also discuss broader sexual assault violence other than rape, such as coercion, unwanted sexual contact, and noncontact unwanted sexual experiences. Coercion includes unwanted sex acts occurring after a person is pressured in nonphysical ways, including being "worn down" by repeatedly being asked for sex or threatened with repercussions in some way (e.g., the victim is told they might be fired, threatened with other blackmail; Black et al., 2011). Unwanted sexual contact includes unwanted sexual touching, such as groping and fondling (Black, 2011). Noncontact unwanted sexual experience includes someone exposing their body parts to a person who does not wish to see them, making a victim look at sexual photos, public harassment that makes the victim feel unsafe (Black et al., 2011). Although men can be (and are) victims of sexual violence, such violence disproportionately affects women (CDC, 2019).

Traumatic events (e.g., sexual assault, natural disaster, death of loved one) can lead to many negative outcomes for the individuals who experience them (American Psychiatric Association; APA, 2013). Such outcomes can include feelings of guilt and shame (Andrews, 
Brewin, Rose, \& Kirk, 2000). For women, sexual assault is related to a myriad of negative mental and physical health symptoms (Black et al., 2011). Compared to women who do not experience sexual assault, those who do experience such trauma report higher prevalence rates of asthma, irritable bowel syndrome, diabetes, frequent headaches, chronic pain, and insomnia (Black et al., 2011). Women who experience sexual assault are more likely to consider their mental and physical health to be poor compared to women without such a history of violence and are at an increased risk for depressive and anxious disorders (Black et al., 2011). Those who experience sexual assault trauma also tend to report higher rates of suicidal ideation than those experiencing other types of traumas (Black et al., 2011; LeBouthillier, McMillan, Thibodeau, \&Asmundson, 2015).

The U.S. has a pervasive rape culture (Buchwald, Fletcher, \& Roth, 1993). Buchwald et al. (1993) describe a rape culture as the encouragement of male sexual aggression such that it perpetuates violence against women. Authors describe such a culture as sexualizing violence which often exists on a continuum experienced by women that escalates from sexualized verbal interaction (e.g. street harassment or "cat calling") to rape itself. A rape culture also adheres to rape myths that even those who experience sexual assault may internalize, leading to feelings of guilt and shame. Rape myths are described as "attitudes and beliefs that are generally false but are widely and persistently held, and that serve to deny and justify male sexual aggression against women" (Lonsway \& Fitzgerald, 1994 pp. 134). Living in and internalizing a rape culture may make the healing process of those who experience sexual assault trauma more difficult compared to those who experience other types of trauma (Moor, 2007). Moor and Farchi (2011) found that individuals who experience sexual assault have greater levels of self-blame compared to those who experience combat stress, car accidents, severe illness, and loss. Excessive blame of the self may make feeling compassion for the self and self-kindness in the face of one's own pain a hard task to undertake, which in turn may make treatment difficult.

\section{Self-Compassion}

Self-compassion is the expression of love, kindness, and understanding toward oneself and is usually exercised in circumstances during which one is likely to engage in harsh selfcriticism (Neff, 2003a; Neff \&Vonk, 2009). Self-compassion is related to the general concept of compassion - being mindful of and touched by the suffering of others, feeling a desire to alleviate that suffering through kindness, and a nonjudgmental understanding of human fallibility (Neff, 2003a). Self-compassion is compassion turned inward - being mindful of and touched by one's own suffering, normalizing one's fallibility, and the desire to alleviate one's own suffering with acts of kindness toward the self (Neff, 2003a).

Self-compassion involves three components: Self-kindness (vs. self-judgment), common humanity (vs. isolation), and mindfulness (vs. overidentification; Neff, 2003a). Neff (2003) explains that when exercising self-compassion, one is generally addressing harmful selfattitudes following a negative situation or in the face of self-attributes or actions that elicit feelings of self-dislike or insecurity. Those who exercise self-compassion are less likely to engage in harsh self-criticism or feel isolated in their pain. They are also more likely to acknowledge their negative emotions and approach their pain with openness and curiosity. When considering their pain, they are less likely to exaggerate or downplay their negative emotions (Neff, 2003a).

Self-compassion is related to less rumination and those higher in self-compassion are less likely to employ coping strategies that rely on avoidance and emotional numbing (Barnard \& Curry, 2011). Furthermore, research has established that self-compassion is significantly and negatively related to certain negative psychological experiences (e.g. depression, anxiety, stress; see meta-analysis by MacBeth \& Gumley, 2012) and that self-compassion inductions can decrease levels of such negative factors (e.g. Neff \& Germer, 2013).

Self-compassion and trauma. According to Germer and Neff (2015), self-compassion can be mapped onto both overarching stress responses and posttraumatic stress symptoms related to traumatic events. The self-kindness component of self-compassion, they claim, is related to the fight stress response and arousal symptoms following trauma. Those lower in self-kindness would, therefore, engage in more self-criticism. The common humanity aspect of self-compassion is related to the flight stress response and the avoidance aspect of trauma. When turning that stress response inward, those lower in common humanity will isolate themselves to flee stress related to traumatic events. Finally, the mindfulness aspect is related 
to the freeze stress response and to intrusive thoughts related to traumatic events. Germer and Neff (2015) claim that those lower in the mindfulness aspect of self-compassion would, theoretically, have a harder time blocking intrusive thoughts related to their traumas.

Correlational research on trait self-compassion has shown thattrait levels of selfcompassion play a significant role in coping with traumatic events (e.g. Thomspon \& Waltz, 2008). It is not uncommon for individuals who have undergone traumatic events to experience self-conscious emotions - feelings of self-disgust, shame, guilt, and anger directed at themselves (e.g. Andrews et al., 2000). Thompson and Waltz (2008) found that participants high in trait self-compassion are less likely to engage in trauma-related avoidance (evading trauma-related stimuli and engaging in emotional numbing) compared to those lower in selfcompassion. Those high in self-compassion may experience a greater willingness to face rather than avoid painful experiences related to trauma (Thompson \& Waltz, 2008). Furthermore, higher levels of the self-judgment component of self-compassionhave been related to more severe posttraumatic stress disorder (PTSD) symptoms (Hoffart, Oktedalen, \& Langkaas, 2015).

In other correlational research, Miron, Orcutt, Hannan, and Thompson (2013) examined levels of self-compassion in individuals who had experienced childhood sexual, emotional, and physical abuse and whether or not these childhood experiences predicted problems with alcohol abuse later in life. A history of emotional abuse indirectly influenced problematic alcohol consumption through low levels of self-compassion, indicating that self-compassion training may be a valuable tool for mitigating some negative behavioral consequences of traumatic experiences. Similarly, Barlow, Turow, and Gerhart (2017) also examined selfcompassion in the context of childhood abuse and found that self-compassion was inversely related to negative trauma appraisals, such as shame and self-blame.

Self-compassion has been related to lower levels of PTSD symptom severity in other types of trauma, also, such as U.S. trauma-exposed soldiers (Dahm et al., 2015; Hiraoka at al., 2015), trauma-exposed college students (Seligowski, Miron, \& Orcutt, 2015), and those who experience natural disasters (Zeller, Yuval, Nitzan-Assayag, \& Bernstein, 2015). More recent research (Bistricky et al., 2017) examining interpersonal trauma (traumas that involve one person intentionally harming another, such as abuse, rape, assault, etc.) has found that those who experienced a greater number or more types of such traumas, who are more avoidantly attached, and who have lower self-compassion may have more severe symptoms related to post trauma stress.

Self-compassion inductions have also become more prevalent in addressing issues related to trauma. Most early literature examining the effects of self-compassion inductions for those who experience trauma focus largely on veterans or general traumatic experiences (Held \& Owens, 2015; Hoffart et al., 2015; Kearney et al., 2015; Klich, 2016). In fact, very little research to date examines self-compassion (correlationally or in the context of interventions) specifically in women who have experienced sexual assault.

Self-compassion inductions may be particularly beneficial for those who experience traumatic events and who identify as women. Some research suggests that women are lower in self-compassion compared to those who identify as men (Neff, 2003a; Neff, Hseih, Dejitthirat, 2005; Raes, 2010; Yarnell, Neff, Davidson, \& Mullarkey, 2018). Women tend to engage in negative, self-critical self-talk more often compared to men (DeVore, 2013), which theoretically supports findings that women are lower in self-compassion. A meta-analysis conducted by Yarnell et al. (2015) examining self-compassion and gender differences in 71 studies revealed that men were slightly higher in self-compassion compared to women with differences between genders being larger among ethnic minorities living in the United States.

Although past research has shown that self-compassion focused practices are beneficial when used in the treatment trauma in general (Held \& Owens, 2015; Hoffart et al., 2015; Klich, 2016), it is thought that women survivors of sexual abuse may experience unique challenges compared to other groups experiencing other types of trauma when engaging in selfcompassion exercises (Boykin et al., 2018; Tesh, Learman, \& Pulliam, 2013). Tesh et al. (2013) provide a theoretical background for appropriateness of self-compassion inductions in the treatment of those who experience intimate partner violence (IPV; i.e. intimidation as well as physical and sexual assault and battery). Authors theorize that mindful self-compassion interventions designed to increase self-kindness, common humanity, and mindfulness would be beneficial in addressing symptoms of shame, self-blame, depression, anxiety, PTSD, and 
suicidality in those who experience IPV.

Boykin et al. (2018) compared women who experienced childhood maltreatment to those who had not experienced such events. Authors found that women survivors of childhood maltreatment (including sexual abuse) tend to actively resist self-kindness and warmth, show a fear of compassion, and exhibit psychological inflexibility during treatment when faced with self-compassion. The more severe the childhood maltreatment, the greater the fear of compassion was for such women (compared to those who experienced minimal or no maltreatment). Close (2013) found that among those who experience sexual assault, higher levels of self-compassion were significantly and negatively related to psychological distress, shame, self-criticism, and trauma-related negative cognitions.

Thought-experiment type research (research in which individuals have not actually experienced an event, but instead are asked to imagine an event) examining self-compassion in the context of sexual assault has shown promising results (Cazeau, 2015). For example, Cazeau (2015) examined benefits of self-compassion inductions compared to a control condition (verbal and serial learning) following an imagined sexual assault scenario. Women who were induced with self-compassion experienced fewer negative effects following the thought experiment compared to women not induced with self-compassion.

Crews, Stolz-Newton, and Grant (2016) found that yoga inductions in group settings for sexual assault survivors increases self-compassion, reduces negative feelings related to trauma, and results in better emotional wellness. Authors claim that positive outcomes of such inductions are a result of increasing feelings of self-kindness, mindfulness, and common humanity (by connecting to other participants).

Small scale qualitative research in therapeutic setting sex plporing the role of selfcompassion in Compassion Focused Therapy (CFT) with women who experience sexual abuse has found that barriers to cultivating self-compassion are related to shame, self-blame, etc. (McLean, Bambling, \& Steindl, 2018). Researchers (McLean et al., 2018) have examined narratives of clients $(n=7)$ undergoing CFT to address issues related to sexual abuse in addition to comments from the clients' counselors $(n=7)$. Transcripts revealed that, among women undergoing CFT after experiencing sexual abuse, self-critical and negative views of the self, shame, self-blame, difficulty in changing self-critical factors, and misinterpretation of what self-compassion is were among the barriers to fostering self-compassion and readiness for effective treatment.

Although these findings illustrate that there may be a unique relationship between selfcompassion and sexual-assault trauma in women in general, research has yet to establish that there is a difference in self-compassion in women who experience sexual assault versus other types of trauma. Past research has not determined whether there is a unique relationship between self-compassion and sexual assault trauma that is different from that of self-compassion and other types of trauma. Much of the aforementioned research examines self-compassion and differing levels of sexual violence or compares women who experience sexual violence to those who do not. More research is needed to determine whether there are differences in self-compassion related to different types of trauma to determine whether sexual violence presents a unique problem for self-compassion in women who experience sexual violence.

\section{The Present Study}

The goal of the current exploratory study was to examine whether different types of traumaspecifically sexual assault versus nonsexual assault trauma - result in differences in selfcompassion (both the total scale subscales) among women. Groups were analyzed both with and without controlling for time. This was done as some research has shown that, for those who experience sexual assault, the passage of time may diminish individuals' negative feelings related to assault (Hunter, 2010; Ovenden, 2012). Finding significant differences in self-compassion based on type of trauma would warrant the need for research on assaultspecific self-compassion interventions.

\section{Method}

\section{Participants}

Participants consisted of a mix of students from a Southeastern university in the United States completing online research for course credit and online volunteers recruited through 
tumbIr, Facebook, and Twitter. Participants were not asked for their location, therefore there is no information on country or region of origin for nonstudent participants. Participants gave consent online after clicking the study link and were assured they could exit the study at any point.

There were originally 323 participants. Of the 323 participants, 113 were removed because they explicitly stated that they did not experience a traumatic event or if the event listed was not a traumatic event (e.g., listing having a very bad dream). Participants who listed family members dying in a nonviolent manner (e.g. due to natural causes) and who did not actually witness these family members die were also excluded as natural-cause death is relatively normal and the process of grief is also normal. Because a goal of the current study was to examine self-compassion in those who experience sexual assault, a predominantly femininegendered issue, participants identifying as male $(N=38)$ were also excluded from analyses.

In total there were 172 participants included in the analyses of the current study. Of these participants, 95 had indicated that they had experienced sexual assault while the other 77 participants listed nonsexual assault potentially traumatic events. The average age of the sample was 23.2 years $(S D=7.17)$. Ethnicity breakdown of participants was: 148 Caucasian, 9 African American, 1 Native American, 1 Asian, 2 Hawaiian/Pacific Islander, 7 Hispanic/Latina, and 4 who chose not to answer or identified as 'Other.' Only 14 participants were nonstudents (e.g. completed the study as part of the advertisement to the general population).

\section{Ethical standards}

The current study was approved by the institution's IRB. Online advertisements asked for participants who had experienced potentially traumatic events and indicated that participants would be asked to write about their traumatic event in addition to completing questionnaires assessing personality characteristics. Consent forms were digital and presented at the beginning of the survey. Participants were informed that they could exit the study at any time they wished without any negative repercussions. Information for mental health services was provided in the consent form as was PI contact information should participants feel distressed. Participants consented by clicking a "Next" button at the bottom of the page. The current research was completed online to protect participant privacy and allow for immediate exit of the study should participants wish to no longer participate (whereas completing a study in a laboratory setting might have made participants feel pressured to continue with the study).

\section{Measures}

Self-Compassion. The original Self-Compassion Scale (SCS; Neff, 2003b) consists of 26 items scored on a 5-point Likert scale ( 1 = Almost never to $5=$ Almost always). The 26 items encompass six subscales (or three competing pairs of subscales). These subscales include self-kindness vs. self-judgment, mindfulness vs. overidentification, and common humanity vs. isolation. Example items include, "I try to be loving towards myself when I'm feeling emotional pain." Internal consistency reliability (Cronbach's alpha) for the total selfcompassion score was .94.

Participants were also asked to list the type of traumatic event they had experienced as well as year of the incident, the age they were when they experienced the incident, and current age. Participants were also asked to list gender and ethnicity.

\section{Results}

An independent samples $t$-test was conducted comparing self-compassion in individuals who experienced sexual assault and nonsexual assault traumatic events. There was a significant difference in self-compassion between those who had experienced sexual assault $(M=72.47, S D=20.24)$ and those who experienced traumatic events that did not involve sexual assault $(M=79.84, S D=19.14) ; t(170)=2.43, p=.02$, Cohen's $d=.36$. For the entire sample, the mean and standard deviation for self-compassion were 75.77 and 20.03 respectively. Results indicated that those who experienced sexual assault had significantly lower levels of self-compassion compared to those who experienced other types of potentially traumatic events.

This difference became marginally significant when controlling for number of years that had passed since the traumatic event $\left(F[1,160]=3.52, p=.06 n p^{2}=.02\right)$. Of the 172 participants 
included in the study, only 71 of nonsexual assault participants listed the approximate date of their potentially traumatic event while 92 of those who experienced sexual assault listed the approximate date of their event. For the entire sample, an average of 12.55 years had passed since the potentially traumatic event described $(S D=8.43)$ with a range of $0-44$ years. For the nonsexual assault group, an average of $18.86(S D=7.64)$ years had passed since their traumatic event with a range of $2-44$ years. For the group of participants who had experienced sexual assault, an average of $10.00(S D=8.16)$ years had passed since their assault with a range of $0-32$ years.

Relationships among subscales and differences in subscale scores of the self-compassion scale between groups were also analyzed. Bivariate correlations indicated that self-kindness, common humanity, and mindfulness were significantly and positively related to one another and were significantly and negatively correlated to self-judgment, isolation, and overidentification. This was true for both participants who had and who had not experienced sexual assault (See Table 1).

Table 1. Group Correlations Among Self-Compassion Subscales

\begin{tabular}{|c|c|c|c|c|c|}
\hline \multicolumn{6}{|c|}{ All participants } \\
\hline & SJ & $\mathrm{I}$ & $\mathrm{O}$ & SK & $\mathrm{CH}$ \\
\hline SJ & -- & & & & \\
\hline I & $.80^{*}$ & -- & & & \\
\hline $\mathrm{O}$ & $.74 *$ & $.79 *$ & -- & & \\
\hline SK & $-.67 *$ & $-.59 *$ & $-.52 *$ & -- & \\
\hline $\mathrm{CH}$ & $-.41 *$ & $-.41 *$ & $-.38 *$ & $.71 *$ & -- \\
\hline M & $-.43 *$ & $-.46^{*}$ & $-.57^{*}$ & $.71 *$ & $.69 *$ \\
\hline \multicolumn{6}{|c|}{ Other trauma } \\
\hline & SJ & I & $\mathrm{O}$ & SK & $\mathrm{CH}$ \\
\hline SJ & -- & & & & \\
\hline I & $.76^{*}$ & -- & & & \\
\hline $\mathrm{O}$ & $.62 *$ & $.75^{*}$ & -- & & \\
\hline SK & $-.67 *$ & $-.58^{*}$ & $-.42 *$ & -- & \\
\hline $\mathrm{CH}$ & $-.45^{*}$ & $-.46^{*}$ & $-.42 *$ & $.72 *$ & -- \\
\hline M & $-.40 *$ & $-.52 *$ & $-.65^{*}$ & $.65^{*}$ & $.68^{*}$ \\
\hline \multicolumn{6}{|c|}{ Sexual assault trauma } \\
\hline & SJ & I & $\mathrm{O}$ & SK & $\mathrm{CH}$ \\
\hline SJ & -- & & & & \\
\hline I & $.81^{*}$ & -- & & & \\
\hline $\mathrm{O}$ & $.81^{*}$ & $.80 *$ & -- & & \\
\hline SK & $-.65^{*}$ & $-.58 *$ & $-.57^{*}$ & -- & \\
\hline $\mathrm{CH}$ & $-.41 *$ & $-.39 *$ & $-.40 *$ & $.73^{*}$ & -- \\
\hline M & $-.47 *$ & $-.44 *$ & $-.55^{*}$ & $.77 *$ & $.70 *$ \\
\hline
\end{tabular}

${ }^{*} p<.01$

$\mathrm{SJ}=$ Self-Judgment, $\mathrm{I}=$ Isolation, $\mathrm{O}=$ Overidentification, $\mathrm{SK}=$ Self-Kindness, $\mathrm{CH}=$ Common Humanity, $M=$ Mindfulness.

A multivariate analysis was conducted to examine differences in self-compassion subscale scores between those who had experienced sexual assault versus nonsexual assault traumas. Type of trauma was, overall, related to significant differences in self-compassion subscale outcomes, $V=.17, F(6,165)=5.79, p<.01 n p^{2}=.17$. Multivariate analyses indicated that those who experienced sexual assault were significantly higher in self-judgment, isolation, and overidentification and marginally significantly lower in self-kindness compared to those who experienced other types of trauma (See Table 2 for multivariate statistics and Table 3 for Ms and SDs). There were no significant differences in common humanity and mindfulness. 
Table 2. Differences Between Groups on Self-Compassion Subscale Scores

\begin{tabular}{llll}
\hline & $F$ & $p$ & $\eta p^{2}$ \\
\hline Self-Judgment & $F(1,165)=9.78$ & $<.01$ & .05 \\
Isolation & $F(1,165)=6.97$ & $<.01$ & .04 \\
Overidentification & $F(1,165)=15.72$ & $<.01$ & .09 \\
Self-Kindness & $F(1,165)=3.84$ & .052 & .02 \\
Common Humanity & $F(1,165)=.11$ & .74 & .00 \\
Mindfulness & $F(1,165)=.01$ & .92 & .00 \\
\hline
\end{tabular}

Table 3. Means and Standard Deviations of Self-Compassion Subscale Scores for Groups

\begin{tabular}{llll}
\hline & Sexual assault trauma & Nonsexual assault trauma & Total \\
& $M(S D)$ & $M(S D)$ & $M(S D)$ \\
\hline Self-Judgment & $17.32(4.78)$ & $15.06(4.61)$ & $16.31(4.83)$ \\
Isolation & $13.43(4.02)$ & $11.79(4.07)$ & $12.70(4.12)$ \\
Overidentification & $14.12(3.77)$ & $11.81(3.84)$ & $12.08(3.96)$ \\
Self-Kindness & $14.02(4.94)$ & $15.42(4.34)$ & $14.65(4.73)$ \\
Common Humanity & $12.52(3.80)$ & $12.37(3.55)$ & $12.44(3.68)$ \\
Mindfulness & $12.79(3.24)$ & $12.74(3.22)$ & $12.77(3.23)$ \\
\hline
\end{tabular}

When controlling for time, there were still overall significant differences between groups in self-compassion subscale outcomes, $V=.17, F(6,165)=5.66, p<.01 \mathrm{np}^{2}=.17$. There remained no significant difference between mindfulness and common humanity. Those who experienced sexual assault remained higher in self-judgment and overidentification, but the differences in isolation became marginally significant and differences in self-kindness became nonsignificant. See Table 4 for multivariate analyses and Table 5 for Ms and SE statistics when controlling for time.

Table 4. Differences Between Groups on Self-Compassion Subscale Scores After Controlling for Time Passed Since Trauma

\begin{tabular}{llll}
\hline & $F$ & $p$ & $\eta p^{2}$ \\
\hline Self-Judgment & $F(1,160)=9.78$ & $<.01$ & .05 \\
Isolation & $F(1,160)=6.97$ & .057 & .02 \\
Overidentification & $F(1,160)=15.72$ & $<.01$ & .07 \\
Self-Kindness & $F(1,160)=3.84$ & .15 & .01 \\
Common Humanity & $F(1,160)=.11$ & .55 & .00 \\
Mindfulness & $F(1,160)=.01$ & .44 & .00 \\
\hline
\end{tabular}

Table 5. Means and Standard Errors of Self-Compassion Subscale Scores for Groups After Controlling for Time Passed Since Trauma

\begin{tabular}{llll}
\hline & Sexual assault trauma & Nonsexual assault trauma & Total \\
& $M(S E)$ & $M(S E)$ & $M(S E)$ \\
\hline Self-Judgment & $17.33(.50)$ & $15.04(.58)$ & $16.18(.37)$ \\
Isolation & $13.36(.42)$ & $12.10(.48)$ & $12.73(.31)$ \\
Overidentification & $14.11(.40)$ & $11.96(.46)$ & $13.03(.30)$ \\
Kindness & $14.05(.49)$ & $15.17(.56)$ & $14.61(.36)$ \\
Common Humanity & $12.42(.39)$ & $12.06(.45)$ & $12.24(.29)$ \\
Mindfulness & $12.80(.33)$ & $12.39(.38)$ & $12.59(.25)$ \\
\hline
\end{tabular}

\section{Discussion}

Past research examining trait self-compassion (e.g. Thompson \& Waltz, 2008) as well as induced self-compassion (Held \& Owens, 2015; Hoffart et al., 2015; Kearney et al., 2015; Klich, 2016)has shown that self-compassion has promising implications in the treatment of those who have experienced potentially traumatic events. Self-compassion may be an important component in recovering from or buffering against negative outcomes related to traumatic experiences. Indeed, researchers such as Thompson and Waltz (2008) have shown that those higher in self-compassion are lower in avoidance related to traumatic events, indicating that having high levels of self-compassion may make one more susceptible to treatment following traumatic events.

Self-compassion and trauma research is a relatively new area of research. Researchers 
have not ventured far beyond pilot and exploratory studies to observe the more nuanced complexities of the relationship of self-compassion with different types of traumatic experiences. Furthermore, no one has examined comparisons of self-compassion among different types of traumatic events to see whether individuals who have experienced certain events have significantly different levels of self-compassion. The goal of the current study was to observe whether individuals who have undergone certain potentially traumatic experiences (i.e. sexual assault and nonsexual assault potential traumas) significantly differ in their levels of self-compassion.

Results indicated that those who experience sexual assault have significantly lower levels of self-compassion than other types of potentially traumatic experiences. When examining the subscales of self-compassion, those who experienced sexual assault were significantly higher in the negative subscales (self-judgment, overidentification, isolation) and marginally significantly lower in self-kindness compared to individuals who experience nonsexual assault potentially traumatic events (when not controlling for time). A rape culture tends to engage in victim-blaming when it comes to sexual assault, which can be internalized by women who experience such traumas (Moor \& Farchi, 2011), therefore it makes sense that participants in the current study who experienced sexual assault would be lower in selfkindness and higher in self-judgment and overidentification compared to participants who experienced other traumas.

It is interesting that, despite there being significant differences in isolation and overidentification, there were no differences between groups in the mindfulness and common humanity subscales. One explanation for this finding could be the types of questions asked in these scales and how they may relate specifically to trauma (versus how their negatively worded counterparts may relate to specific types of trauma or trauma in general). For example, the questions for common humanity are:

3. When things are going badly for me, I see the difficulties as part of life that every one goes through.

7. When I'm down and out, I remind myself that there are lots of other people in the world feeling like I am.

10. When I feel inadequate in some way, I try to remind myself that feelings of inadequacy are shared by most people.

15. I try to see my failings as part of the human condition.

The counterpart questions for the isolation subscale (the opposite of common humanity) are:

4. When I think about my inadequacies, it tends to make me feel more separate and cutoff from the rest of the world.

13. When I'm feeling down, I tend to feel like most other people are probably happier than I am.

18. When I'm really struggling, I tend to feel like other people must be having an easier time of it.

25. When I fail at something that's important to me, I tend to feel alone in my failure.

Those experiencing nonsexual assault traumas may not feel as much isolation compared to sexual assault trauma because such events maybe more normalized. Even though experiencing traumatic events may be viewed as a rare phenomenon, events not involving sexual assault may be viewed as more "acceptable" or at least less shameful compared to sexual assault. Society may be less likely to blame victims of nonsexual traumas (see information on rape culture and rape myths in introduction), meaning that such individuals may be less likely to blame themselves compared to those who experience sexual assault. The isolation questions may especially resonate with those who experience sexual assault, a traumatic event prone to blaming victims and eliciting greater feelings of shame (Moor \& Farchi, 2011).The isolation scale may be where we see the unique influence of type of trauma on self-compassion. All people experiencing trauma may feel that others have an easier time (hence no differences between groups in common humanity), but those experiencing sexual assault may feel especially isolated (even compared to other types of trauma). People who experience sexual assault may agree more strongly with items in the isolation scale compared to people with other traumas even when all participants feel isolated. However, the positively- 
worded common humanity items may not be nuanced enough when examining the effects of different types of trauma on self-compassion. Statements involving seeing difficulties as things everyone goes through, or that lots of people in the world feel like the participant does, or that their experiences are part of the human condition may elicit equal levels of disagreement among participants regardless of type of trauma. Future research using the self-compassion scale in trauma studies may benefit from exploring this issue further and perhaps even modifying the scale to be more specific to trauma. For example, instead of stating "When things are going badly for me, I see the difficulties as part of life that everyone goes through," researchers might reword the items to include trauma-relevant experiences "When I'm suffering or experiencing setbacks because of my traumatic experience, I see my difficulties as part of the process of recovery that all people who experience trauma go through." This type of rewording could, conceivably, be truer for those who experience nonsexual assault traumas and less true for those who experience sexual assault traumas as sexual assault can be related to greater feelings of guilt and shame (Moor \& Farchi, 2011), and therefore, isolation. The same issue may also explain why there are differences in overidentification but not mindfulness. Overidentification items may allow for a more nuanced view between different types of trauma, while mindfulness items may be too generalized to do so.

Analyses controlling for time were also conducted as some research has shown that time may reduce individuals' negative feelings related to assault (Hunter, 2010; Ovenden, 2012). The difference became marginally significant at $p=.06$ when controlling for number of years passed since the event. When examining subscales after controlling for time passed since assault, differences generally stayed the same excepting that differences in isolation became marginally significant and differences in self-kindness went from marginally significant to nonsignificant. Past research on transformative narratives has shown that, over time, people who experience sexual assault may shift from viewing themselves as a victim of sexual assault to a more empowered view of themselves as a survivor of sexual assault (Hunter, 2010). This transformation may be influenced by increased awareness of prevalence of assault which leads individuals to understand that they are not alone or to blame for their experience. This may explain why differences between groups in isolation and self-kindness changed after controlling for time. Currently, only one study has examined the differences in self-compassion between individuals who consider themselves a survivor, victim, or neither (survivor nor victim) of sexual assault (Williamson \& Serna, 2017). There were no differences in self-compassion between labels; however, the authors did not examine differences in selfcompassion subscale outcomes between labels. It should also be noted that some participants were not included in analyses controlling for time as they did not list when their traumatic event happened nor did they list the age of their event. Differences in outcomes compared to analyses not controlling for time may very well be due to the exclusion of the participants who did not list time.

One limitation to the current study is that it is not known whether the self-compassion scores reflect changes in self-compassion as a result of traumatic events or whether the observed levels of self-compassion are stable despite traumatic events. It is not known whether sexual assault actually decreases levels of self-compassion more drastically compared to other types of potential traumas, or whether the observed levels of self-compassion existed before traumatic events.

The fact that PTSD symptoms or other negative outcomes such as shame and self-blame were not measured is another limitation. However, the relationship between self-compassion, PTSD, and other symptoms related to trauma have been well-established in past studies (e.g. Thompson \& Waltz, 2008). The current study was merely an exploratory study to examine whether specific types of trauma - sexual assault compared to nonsexual assault - are related to different levels of self-compassion.

Not collecting information on place of origin may be another limitation as a small number of participants were recruited online and not from schools. Different cultures may have different views and societal treatment of those who experience sexual assault compared to the United States. However, it should be noted that even if data were collected only from students, participants still may have come from other countries.

Inductions tailored specifically for sexual assault may be particularly useful considering that findings of the current study indicate that those who experience sexual assaulthave worse 
self-compassion outcomes compared to those who experience other traumas. This may be especially true if such interventions target self-judgment, self-kindness, isolation, and overidentification. Past research has shown that self-compassion inductions are useful for addressing factors related to trauma in general. Studies that have experimentally manipulated self-compassion involve several different types of inductions to increase selfcompassion, including Compassionate Mind Training paired with Cognitive Behavior Therapy (Beaumont, Galpin, \& Jenkins, 2012), using self-compassion workbooks to address traumarelated guilt in homeless veterans (Held \& Owens, 2015), and loving kindness meditation to address PTSD symptoms(Kearney et al., 2013).Although some past research has examined self-compassion oriented interventions specifically in women who experience IPV and childhood maltreatment (Crews et al., 2016), no research has supported the idea that there are distinct self-compassion outcomes among women following sexual assault compared to other types of trauma. The current study supports theoretical papers such as that of Tesh et al. (2013) proposing that there is a need for self-compassion interventions designed specifically to help women who experience sexual assault.

Future research might modify existing self-compassion inductions used in traumatic contexts to specifically address sexual assault related outcomes. These inductions could be provided as supplements to therapies used to treat trauma. Furthermore, compassion-based therapies (e.g. Beaumont et al., 2012; Kearney et al., 2013) such as Loving Kindness Meditation and Compassionate Mind Training might be adapted to specifically treat sexual assault trauma by focusing more on self-judgment and self-kindness, overidentification, and isolation as these are where significant differences were found in the current study. Such past research, when coupled with the findings of the current study, illustrate the possible utility of using self-compassion interventions to address negative symptoms in those who experience sexual assault.

Finding that aspects of self-compassion may be significantly different based on type of trauma have implications for trauma treatment. The current study indicates that perhaps not all traumas are equal in their relationship with self-compassion. The translational implications of the current study indicate a need for further research on the benefits of self-compassion for different types of trauma and a more in-depth look at what specific negative posttraumatic outcomes (e.g. self-blame, shame, guilt, depression, anxiety) are best addressed by selfcompassion inductions.

\section{References}

American Psychiatric Association (2013). Diagnostic and statistical manual of mental disorders (5th ed.). Arlington, VA: American Psychiatric Publishing.

Andrews, B., Brewin, C., Rose, S., \& Kirk, M. (2000). Predicting PTSD symptoms in victims of violent crime: The role of shame, anger, and childhood abuse. Journal of Abnormal Psychology, 109, 69-73.

Barlow, M.R., Turow, R.E.G., \& Gerhart, J. (2017). Trauma appraisals, emotion regulation difficulties, and self-compassion predict posttraumatic stress symptoms following childhood abuse. Child Abuse \& Neglect, 65, 37-47. DOI: http//dx.doi.org/10.1016/j.chiabu.2017.01.006

Barnard, L. K., \& Curry, J. F. (2011). Self-compassion: Conceptualizations, correlates, \& interventions. Review of General Psychology, 15 (4), 289-303.

Basile, K.C, Smith, S.G., Breidling, M.J., Black, M.C., \&Mahendra, R.R. (2014). Sexual Violence Surveillance: Uniform Definitions and Recommended Data Elements, Version 2.0. Atlanta (GA): National Center for Injury Prevention and Control, Centers for Disease Control and Prevention.

Beaumont, E., Galpin, A., \& Jenkins, P. (2012). Being kinder to myself: A prospective comparative study, exploring post-trauma therapy outcome measures, for two groups of clients, receiving either Cognitive Behaviour Therapy or Cognitive Behaviour Therapy and Compassionate Mind Training. Counselling Psychology Review, 27, 31-43.

Bistricky, S.L., Gallagher, M.W., Roberts, C.M.M, Ferris, L., Gonzalez, A.J., Wetterneck, C.T. (2017). Frequency of interpersonal trauma types, avoidant attachment, self-compassion, and interpersonal competence: A model of persisting posttraumatic symptoms. Journal of Aggression, Maltreatment, \& Trauma, 26(6), 608-625, DOI: 10.1080/10926771.2017.1322657

Black, Basile, Breiding, Smith, Walters, Merrick, ... \& Stevens (2011, March 5). The national intimate partner and sexual violence survey: 2010 summary report. Retrieved from the Centers for Disease Control and 
Prevention, National Center for Injury Prevention and Control: http://www.cdc.gov/violenceprevention /pdf/nisvs_report2010-a.pdf

Boykin, D. M., Himmerich, S. J., Pinciotti, C. M., Miller, L. M., Miron, L. R., \& Orcutt, H. K. (2018). Barriers to self-compassion for female survivors of childhood maltreatment: the roles of fear of self-compassion and psychological inflexibility. Child abuse \& neglect, 76, 216-224.

Buchwald, E., Fletcher, P., \& Roth, M. (1993). Transforming a rape culture. Minneapolis, MN: Milkweed Editions.

Cazeau, S. (2015). Taking the victim out of sexual assault: The effect of self-compassion on sexual assault survivors (Master's thesis). Retrieved from http://digitalcommons.unf.edu/etd/574/

Centers for Disease Control and Prevention (2019, March 12). Preventing Sexual Violence. Retrieved from https://www.cdc.gov/violenceprevention/sexualviolence/fastfact.html

Crews, D. A., Stolz-Newton, M., \& Grant, N. S. (2016). The use of yoga to build self-compassion as a healing method for survivors of sexual violence. Journal of Religion \& Spirituality in Social Work: Social Thought, 35(3), 139-156.

Close, A. (2013). Self-compassion and recovery from sexual assault (Order No. 3665732). Available from ProQuest Dissertations \& Theses Global: Social Sciences. (1631129544).

Dahm, K. A., Meyer, E. C., Neff, K. D., Kimbrel, N. A., Gulliver, S. B., \&Morissette, S. B. (2015). Mindfulness, self-compassion, posttraumatic stress disorder symptoms, and functional disability in U.S. Iraq and Afghanistan war veterans. Journal of Traumatic Stress, 28(5), 460-464. doi:http://dx.doi.org.er.lib.kstate.edu/10.1002/jts.22045

DeVore, R. (2013). Analysis of gender differences in self-statements and mood disorders. McNair Scholars Research Journal, 9, 1-7.

Germer, C. K., \& Neff, K. D. (2015). Cultivating self-compassion in trauma survivors. In V. M. Follette, J. Briere, D. Rozelle, J. W. Hopper, \& D. I. Rome (Eds.), Mindfulness-oriented interventions for trauma: Integrating contemplative practices (pp. 43-58). New York, NY, US: The Guilford Press.

Held, P., \& Owens, G. P. (2015). Effects of self-compassion workbook training on trauma-related guilt in a sample of homeless veterans: A pilot study. Journal of Clinical Psychology, 71(6), 513.

Hiraoka, R., Meyer, E. C., Kimbrel, N. A., DeBeer, B. B., Gulliver, S. B., \&Morissette, S. B. (2015). Selfcompassion as a prospective predictor of PTSD symptom severity among trauma-exposed U.S. Iraq and Afghanistan war veterans. Journal of Traumatic Stress, 28(2), 127-133. doi:http://dx.doi.org.er.lib.kstate.edu/10.1002/jts.21995

Hoffart, A., Oktedalen, T., \&Langkaas, T.F. (2015). Self-compassion influences PTSD symptoms in the process of change in trauma-focused cognitive behavioral therapies: A study of within-person processes. Frontiers in Psychology, 6, 1273-1284. DOI: 10.3389/psycg.2015.01273

Hunter, S. V. (2010). Evolving narratives about childhood sexual abuse: Challenging the dominance of the victim and survivor paradigm. Australian and New Zealand Journal of Family Therapy, 31(2), 176-190.

Kearney, D.J., Malte, C.A., McManus, C., Martinez, M.E., Felleman, B., \& Simpson, T.L. (2013). Lovingkindness mediation for posttraumatic stress disorder: A pilot study. Journal of Traumatic Stress, 26, 426434. doi: $10.1002 /$ jts. 21832

Klich, U. (2016). Clinical use of self-compassion within mindfulness-based biofeedback in the treatment of veterans and spouses: Acase study. Biofeedback, 44(3), 138-144.

LeBouthillier, D. M., McMillan, K. A., Thibodeau, M. A., \& Asmundson, G. J. G. (2015). Types and number of traumas associated with suicidal ideation and suicide attempts in PTSD: Findings from a U.S. nationally representative sample. Journal of Traumatic Stress, 28(3), 183-190. doi:http://dx.doi.org.er.lib.kstate.edu/10.1002/jts.22010

Lonsway, K. A., \& Fitzgerald, L. F. (1994). Rape myths: In review. Psychology of Women Quarterly, 18, $133-164$.

MacBeth, A., \&Gumley, A. (2012). Exploring compassion: A meta-analysis of the association between selfcompassion and psychopathology. Clinical Psychology Review, 32, 545-552.

McLean, L., Bambling, M., \& Steindl, S. R. (2018). Perspectives on self-compassion from adult female survivors of sexual abuse and the counselors who work with them. Journal of Interpersonal Violence. https://doi.org/10.1177/0886260518793975

Miron, L. R., Orcutt, H. K., Hannan, S. M., \& Thompson, K. L. (2014). Childhood abuse and problematic alcohol use in college females: The role of self-compassion. Self and Identity, 13(3), 364-379. http://dx.doi.org/10.1080/15298868.2013.836131 
Moor, A. (2007). When recounting the traumatic memories is not enough: Treating persistent selfdevaluation associated with rape and victim-blaming rape myths. Women \& Therapy, 30(1-2), 19-33. doi:http://dx.doi.org.er.lib.k-state.edu/10.1300/J015v30n01_02

Moor, A., \& Farchi, M. (2011). Is rape-related self-blame distinct from other post traumatic attributions of blame? A comparison of severity and implications for treatment. Women \& Therapy, 34(4), 447. Retrieved from http://search.proquest.com.er.lib.k-state.edu/docview/898724076?accountid=11789

Neff, K. D. (2003a). Self-compassion: An alternative conceptualization of a healthy attitude toward oneself. Self and Identity, 2, 85-102. DOI: 10.1080/15298860390129863

Neff, K. D. (2003b). The development and validation of a scale to measure self-compassion. Self and Identity, 2, 223-250. DOI: 10.1080/15298860390209035

Neff, K. D., \& Germer, C. K. (2013). A pilot study and randomized controlled trial of the Mindful SelfCompassion program. Journal of Clinical Psychology, 69(1), 28-44.

Neff, K. D., Hseih, Y., \& Dejitthirat, K. (2005). Self-compassion, achievement goals, and coping with academic failure. Self and Identity, 4, 263-287.

Neff, K.D. \& Vonk, R. (2009). Self-compassion versus global self-esteem: Two different ways of relating to oneself. Journal of Personality, 77, 23-50.

Ovenden, G. (2012). Young women's management of victim and survivor identities. Culture, Health \& Sexuality, 14(8), 941-954

Raes, F. (2010). Rumination and worry as mediators of the relationship between self-compassion and depression and anxiety. Personality and Individual Differences, 48,757-761.

Seligowski, A. V., Miron, L. R., \& Orcutt, H. K. (2015). Relations among self-compassion, PTSD symptoms, and psychological health in a trauma-exposed sample. Mindfulness, 6(5), 1033-1041. doi: http://dx.doi.org/ $10.1007 / \mathrm{s} 12671-014-0351-\mathrm{x}$

Tesh, M., Learman, J., \& Pulliam, R. M. (2013). Mindful self-compassion strategies for survivors of intimate partner abuse. Mindfulness, 6 (2),192-201.

Thompson, B. L., Waltz, J. (2008) Self-compassion and PTSD symptom severity. Journal of Traumatic Stress, 21, 556-558. doi: 10.1002/jts.20374

Williamson, J., \& Serna, K. (2017). Reconsidering forced labels: Outcomes of sexual assault survivors versus victims (and those who choose neither). Violence Against Women, 24(16), 668-683. doi/10.1177/1077801217711268

Yarnell, L. M., Neff, K. D., Davidson, O. A., \& Mullarkey, M. (2018). Gender differences in self-compassion: Examining the role of gender role orientation. Mindfulness, 1-17.

Yarnell, L. M., Stafford, R. E., Neff, K. D., Reilly, E. D., Knox, M. C. \& Mullarkey, M. (2015). Meta-analysis of gender differences in self-compassion. Self and Identity, 14(5), 1-22. http://dx.doi.org/10.1080 /15298868.2015.1029966

Zeller, M., Yuval, K., Nitzan-assayag, Y., \& Bernstein, A. (2015). Self-compassion in recovery following potentially traumatic stress: Longitudinal study of at-risk youth. Journal of Abnormal Child Psychology, 43(4), 645-653. doi:http://dx.doi.org/10.1007/s10802-014-9937 